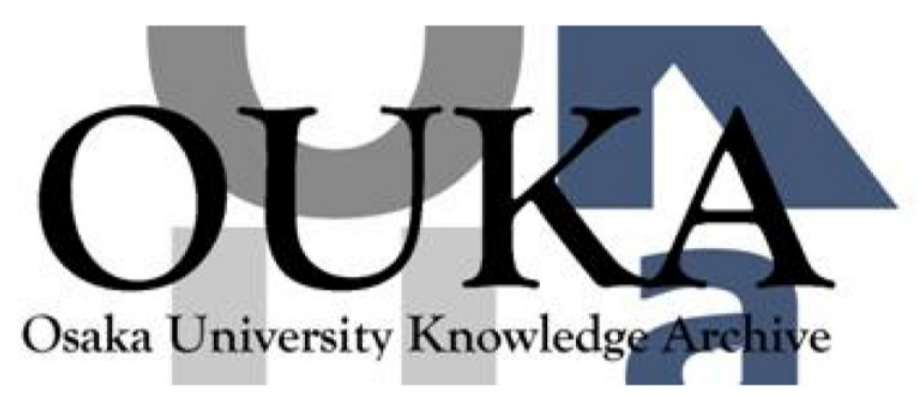

\begin{tabular}{|c|l|}
\hline Title & $\begin{array}{l}\text { Stacking-fault structure explains unusual } \\
\text { elasticity of nanocrystalline diamonds }\end{array}$ \\
\hline Author(s) & Tanei, H.; Tanigaki, K. ; Kusakabe, K. et al. \\
\hline Citation & $\begin{array}{l}\text { Applied Physics Letters. 94(4) p. 041914-1- } \\
\text { p.041914-3 }\end{array}$ \\
\hline Issue Date & $2009-01-26$ \\
\hline oaire:version & VoR \\
\hline URL & $\begin{array}{l}\text { https://hdl. handle. net/11094/84210 } \\
\text { This article may be down loaded for personal use } \\
\text { only. Any other use requires prior permission } \\
\text { of the author and AIP Publishing. This art icle } \\
\text { appeared in Applied Physics Let ters, 94(4), } \\
041914 \text { (2009) and may be found at } \\
\text { https://doi.org/10.1063/1.3077124. }\end{array}$ \\
\hline rights \\
\hline Note & \begin{tabular}{l}
$\mid$ \\
\hline
\end{tabular} \\
\hline
\end{tabular}

Osaka University Knowledge Archive : OUKA

https://ir. Library. osaka-u. ac. jp/

Osaka University 


\title{
Stacking-fault structure explains unusual elasticity of nanocrystalline diamonds
}

\author{
H. Tanei, K. Tanigaki, K. Kusakabe, H. Ogi, ${ }^{a)}$ N. Nakamura, and M. Hirao \\ Graduate School of Engineering Science, Osaka University, Toyonaka, Osaka 560-8531, Japan
}

(Received 21 November 2008; accepted 7 January 2009; published online 29 January 2009)

\begin{abstract}
This letter reveals that unusual elasticity of nanocrystalline diamond is consistently explained by stacking fault inside the diamond grains instead of the graphitic plate inclusion, which was only possible mechanism. Ab initio calculation shows that stacking fault introduced in the diamond structure behaves as graphitic $s p^{2}$ bonds, and the elastic constants calculated from the strain-energy relationship agree with the acoustic measurements. (c) 2009 American Institute of Physics. [DOI: $10.1063 / 1.3077124$ ]
\end{abstract}

Nanocrystalline diamond (NCD) thin films deposited by chemical vapor deposition (CVD) with a small amount of nitrogen gas show highly smooth surfaces, and they are candidates for high-efficiency acoustic and electronic devices. ${ }^{1-3}$ However, incorporated nitrogen gas generates nanodefects and various precipitates, deteriorating attractive mechanical properties of diamond, which have great influence on the lifetime of the devices. Thus, the relationship between the microstructure of NCD and mechanical properties remains a central issue. We previously investigated the influence of nitrogen gas on the elastic properties. ${ }^{4}$ Our study showed that the increase in the nitrogen gas concentration decreases the diagonal elastic constants $C_{11}, C_{33}$, and $C_{66}$ of NCD, while it significantly increases the off-diagonal components $C_{12}$ and $C_{13}$. This behavior was analyzed by a macroscopic model, considering small amount of inclusions in the diamond structure. As the possible inclusion, we assumed microcracks, diamond-like carbon, and monocrystal graphite. Only the monocrystal graphite inclusion can explain the significant increase in the off-diagonal components. ${ }^{4}$ Such a microstructure is consistent with the studies by Birrell et al., ${ }^{5,6}$ which suggest that NCD structure consists of small amount of $s p^{2}$-bonded carbons near boundaries of $s p^{3}$-bonded crystalcarbon grains. However, the monocrystal-graphite plate will not appear in the all grain boundaries and no study succeeded in observing them so far. Thus, the detailed mechanism of the unusual elastic property of NCD remains unknown and more appropriate microstructure model has been searched.

In this study, we show evidence of the significant contribution of stacking faults in diamond structure to the unusual elastic behavior using ab initio calculations. The periodic stacking faults are introduced in the [111] stacking sequence of diamond, and the elastic constants are calculated from the energy-strain relationship. Then, the Hill-averaging approximation is made to simulate a diamond structure involving randomly oriented stacking-fault phases, which can be a model for the NCD. The results are compared with acoustic measurements we originally developed. The bond structure is discussed using the partial density of states (PDOS) of individual atoms.

We measure the elastic constants of three kinds of NCD thin films considering them as isotropic materials. X-ray dif-

${ }^{a)}$ Electronic mail: ogi@me.es.osaka-u.ac.jp. fraction patterns indicate insignificant texture of the NCD specimens, allowing the isotropic assumption. NCD thin films are deposited by the hot-filament CVD method with various concentrations of nitrogen gas in the flow. Raman spectroscopy clearly shows that the nitrogen gas develops the $s p^{2}$-bonded region. We here define the carbon atoms, which are prevented from $s p^{3}$-bond formation by the incorporated nitrogen as non-s $p^{3}$ bonds (such as $s p^{2}$-bonded carbons). The elastic constants are determined by the resonant ultrasound spectroscopy ${ }^{7}$ and Brillouin oscillations. ${ }^{8}$ Figure 1 shows the dependence of Young's modulus $E$, shear modulus $G$, and Poisson's ratio $\nu$ on the flow rate of nitrogen gas. As the amount of nitrogen gas increases, $E$ and $G$ decrease by $20 \%-30 \%$, while $\nu$ significantly increases by more than $200 \%$. Such a large change in $\nu$ cannot be achieved by usual inclusions such as defects and amorphous impurities.

For example, we calculate the macroscopic elastic constants using the micromechanics calculation ${ }^{9,10}$ and Hillaveraging method, ${ }^{11}$ and compare them with measurements in Fig. 1. The NCD structure is considered as a composite consisting of isotropic diamond matrix and randomly oriented pancake-shaped thin inclusions. The elastic constants of such a model are calculated with changing the volume fraction of the inclusion. The nitrogen gas decreases the
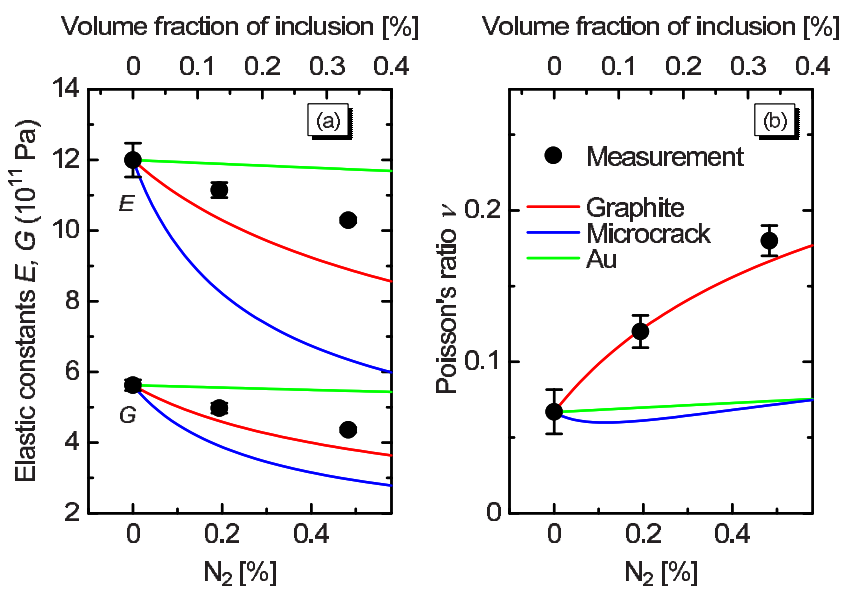

FIG. 1. (Color online) Measured dependence of (a) Young's modulus $E$ and shear modulus $G$ and (b) Poisson's ratio $\nu$ on the flow rate of nitrogen gas (solid marks). The solid lines represent the dependence of (a) $E$ and $G$ and (b) $\nu$ on the volume fraction of thin-plate inclusions with the aspect ratio of 1000 calculated by the micromechanics calculation. 
grain size, causing the increase in the volume fraction of grain boundaries, where the non- $s p^{3}$ bonds appear. ${ }^{5,6} \mathrm{Be}$ cause we simulate the non- $s p^{3}$ regions by the inclusions, the increase in the volume fraction of the inclusion corresponds to the increase in the amount of nitrogen gas. For the non-sp $p^{3}$ region, we assume three inclusions: (i) monocrystal graphite $(\nu=0.34),{ }^{12}$ (ii) incompressible air $(\nu=0.50)$, and (iii) isotropic $\mathrm{Au}(\nu=0.43){ }^{13}$ We adopt Au because it shows larger Poisson's ratio than that of diamond by a factor of 6.3. Despite the large Poisson's ratio, air and Au inclusions fail to explain the large increase (more than twice) in $\nu$. As demonstrated in Fig. 1, only monocrystal graphite inclusion can consistently explain it.

It would be, however, unrealistic that all grain boundaries are composed of monocrystal graphitic thin plates, and such a microstructure has never been observed. Then, we propose a microstructure, which contains graphitic bonds in $s p^{3}$-bonded diamond, that is the stacking fault. This structure can be an essential model for non- $s p^{3}$ bonds because there are various distorted regions in NCD thin films, and their bonds are certainly different from that by $s p^{3}$. We perform $a b$ initio calculations using the PWSCF code, ${ }^{14}$ which is based on a plane-wave pseudopotential approach to the densityfunctional theory. ${ }^{15,16}$ The exchange correlation functional is treated using the local density approximation. The energy cutoff value of the plane-wave expansion for the wave function is $40 \mathrm{Ry}$, and that of the charge density is $200 \mathrm{Ry}$. The $k$-point mesh for the calculations is $8 \times 8 \times 8$. These calculation conditions give less than $0.1 \%$ fluctuations in the lattice parameters and elastic constants.

We then consider the periodic stacking-fault structure. A monocrystal bulk diamond has six atoms in the primitive cell along the [111] direction as aa'bb'cc'. In order to construct the stacking fault, the additional layers of aa' are inserted, or cc' layers are removed from periodic stacking sequence of diamond (111). Thus, we construct four types of periodic stacking-fault structures: (i) ABCA, (ii) ABCAB, (iii) $\mathrm{ABCABCA}$, and (iv) $\mathrm{ABCABCAB}$ (for the simplicity, set of layers aa', bb', and cc' are denoted as A, B, and C, respectively). In the optimization, the supercell is fixed to remain hexagonal symmetry, and each layer is constrained not to slide transversely in order to intentionally construct non-s $p^{3}$-bonded stacking fault in the $s p^{3}$ structure. Because (111) surfaces of diamond could cause rearrangement, ${ }^{17-19}$ carbon atoms facing the stacking fault could have been reconstructed without any external restriction. However, we ignore this rearrangement because of three reasons: (i) The purpose of this study is to clarify the influence of the remaining stacking fault on the elastic constants, not to determine a stable defective structure. The surface rearrangement will relax atoms near the stacking fault, making their influence on the elastic property less significant. (ii) Even if the surface relaxation vanishes the stacking fault and develops the $s p^{3}$-bonded regions, such a region is involved in the bulkdiamond behavior and out of interest. (iii) There are many impurities and defects at grain boundaries of real NCD microstructure, and they somehow contribute to restrict movement of atoms near the defects. Because it is unrealistic to simulate such complicated real microstructure by $a b$ initio calculation, the stacking faults can be an important possible model when it consistently explains the unusual elasticity of NCD.
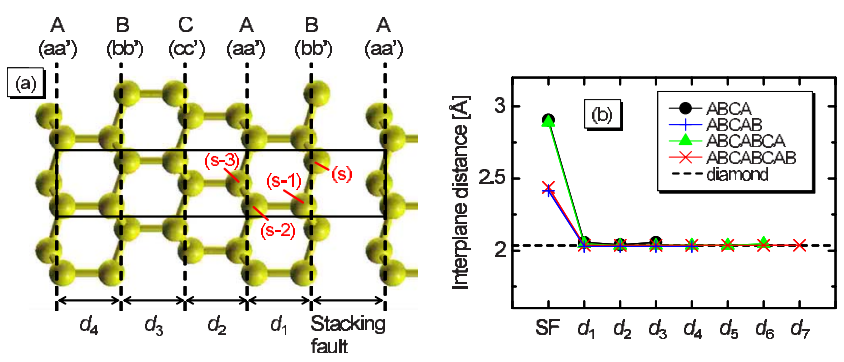

FIG. 2. (Color online) (a) Optimized stacking-fault structure of ABCAB The solid box represents a unit cell. (b) Change in the interplane distances of optimized structures.

Before the optimization, the thickness of the stackingfault layer was set to the interplane distance of the bulk diamond. Figure 2 shows an optimized ABCAB supercell and the interatomic distance in the out-of-plane direction (along [111] direction) individually. The structure relaxation changes the interplane distance for inner atoms only by $1 \%$ from that of the bulk diamond, while it increases thickness of the stacking fault by $20 \%-40 \%$.

We then calculate the elastic constants of these structures using the harmonic-lattice approximation. ${ }^{20}$ The total energy is calculated by changing the strain from $-1 \%$ to $1 \%$ by a $0.2 \%$ step, and the quadratic function is fitted to the energystrain plot. With this method, we determine the five elastic constants $c_{11}, c_{33}, c_{13}, c_{12}$, and $c_{44}$ with hexagonal symmetry. The details to determine the elastic constants appear in the supporting material. ${ }^{21}$ Figure 3 (a) shows the relationship between the calculated elastic constants and the average of the interplane distance. Because the increase in the average interplane distance is mainly caused by the increase in the thickness of the stacking fault [Fig. 2(b)], the increase in the average interplane distance corresponds to the increase in the volume fraction of defects, and then shows a positive correlation with the amount of nitrogen gas. In Fig. 3(a), as the interplane distance increases, the out-of-plane elastic moduli $c_{33}$ and $c_{44}$ decrease, indicating that the atoms facing stacking faults are not bonded tightly in the out-of-plane direction. On the other hand, the off-diagonal stiffness $c_{13}$ increases with the increase in the average interplane distance.

Because the measured NCD shows nearly isotropic polycrystalline structure, we make the direction averaging on the calculated elastic constants using the Hill-averaging method
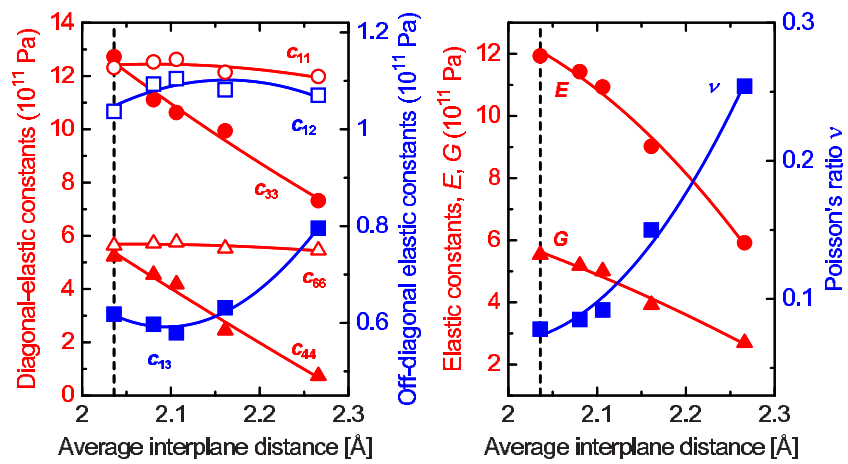

FIG. 3. (Color online) (a) Dependence of the calculated elastic constants of the hexagonal-symmetry diamonds with stacking faults on the average interplane distance and (b) that of the elastic constants of aggregated structure obtained by the Hill-averaging approximation. The broken lines represent the calculated interplane distance of the defect-free diamond along [111] direction. 

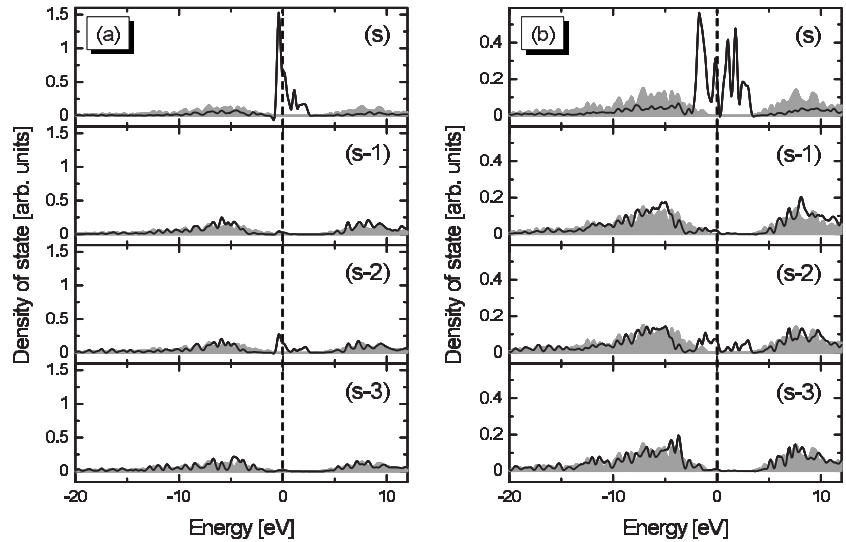

FIG. 4. PDOS of the $2 p_{z}$ orbital of carbon atoms in the (a) ABCABCA and (b) $\mathrm{ABCABCAB}$ structures. The zero energy is set to the Fermi level. The index $(s)$ represents the atom at the stacking fault, and the $(s-i)$ indexes represent the $i$ th subsequent atoms (see Fig. 2). The filled areas represent the PDOS of the bulk diamond.

to construct randomly oriented stacking faults. Figure 3(b) shows the results. The increase in the average interplane distance decreases $E$ and $G$, and significantly increases $\nu$. Therefore, the atomic-scale model proposed here reproduces the significant increase in $\nu$. However, the average interplane distance cannot quantitatively reproduce the changes in the elastic moduli. For example, the interplane distance of $2.2 \AA$ causes the increase in $\nu$ up to 0.18 , which is close to measured $\nu$ at the nitrogen flow rate of $0.5 \%$. However, this distance causes $E$ and $G$ smaller than the measurements. Real NCD will involve many types of stacking-fault structures not only (111) stacking sequence but also (001) and (110). Also, any other structures such as impurities and nanodefects exist. These complicated structures will make the quantitative comparison with measurement difficult. The calculated elastic trend is, however, well consistent with that of the measurement, indicating that the non- $s p^{3}$ structure in $s p^{3}$ bonds has dominant influence on the elastic properties of NCD.

Figure 4 shows the PDOS profiles of the $2 p_{z}$ orbital of the carbon atom facing the stacking faults $(s)$ and subsequent three inner atoms $(s-1),(s-2)$, and $(s-3)$ indicated in Fig. 2(a). For the inner atoms, their PDOS shows a similar distribution to that of the bulk diamond. For the stacking-fault atom, however, large peaks appear near the Fermi level. In general, when an atom shows dangling bonds, such as graphite, PDOS peaks are generated around the Fermi level. Thus, the PDOS distribution indicates non- $s p^{3}$ bonds along the [111] direction between the stacking-fault atoms. This result is consistent with the micromechanics calculation that thin graphitic (non- $s p^{3}$ bonded) region is required to explain the unusual increase in Poisson's ratio (or off-diagonal stiffness).

In summary, our $a b$ initio calculations predict a diamond model with randomly oriented stacking faults, which consistently explains the acoustic measurements and micromechanics calculation. The elastic constants of such a diamond structure reconstruct the unusual elastic behavior of NCD, where Young's modulus and shear modulus decrease, and Poisson's ratio significantly increases as the increase in the volume fraction of the defects. The stacking-fault model presented here is a representative case and it provides us with the essential behavior of the non- $s p^{3}$ bonds on the stiffness.

${ }^{1}$ O. A. Williams, S. Curat, J. Gerbi, D. M. Gruen, and R. B. Jackman, Appl. Phys. Lett. 85, 1680 (2004).

${ }^{2}$ P. Achatz, O. A. Williams, P. Bruno, D. M. Gruen, J. A. Garrido, and M. Stutzmann, Phys. Rev. B 74, 155429 (2006).

${ }^{3}$ F. Bénédic, M. B. Assouar, P. Kirsch, D. Monéger, O. Brinza, O. Elmazria,

P. Alnot, and A. Gicquel, Diamond Relat. Mater. 17, 804 (2008).

${ }^{4}$ H. Tanei, N. Nakamura, H. Ogi, M. Hirao, and R. Ikeda, Phys. Rev. Lett. 100, 016804 (2008)

${ }^{5}$ J. Birrell, J. A. Carlisle, O. Auciello, D. M. Gruen, and J. M. Gibson, Appl. Phys. Lett. 81, 2235 (2002).

${ }^{6}$ J. Birrell, J. E. Gerbi, O. Auciello, J. M. Gibson, J. Johnson, and J. A. Carlisle, Diamond Relat. Mater. 14, 86 (2005).

${ }^{7}$ N. Nakamura, H. Ogi, M. Hirao, and T. Ono, Appl. Phys. Lett. 86, 111918 (2005)

${ }^{8}$ H. Ogi, T. Shagawa, N. Nakamura, M. Hirao, H. Odaka, and N. Kihara, Phys. Rev. B 78, 134204 (2008).

${ }^{9}$ H. Ogi, G. Shimoike, M. Hirao, K. Takashima, and Y. Higo, J. Appl. Phys. 91, 4857 (2002).

${ }^{10}$ N. Nakamura, H. Ogi, and M. Hirao, Acta Mater. 52, 765 (2004).

${ }^{11}$ R. Hill, J. Mech. Phys. Solids 5, 229 (1957).

${ }^{12}$ O. L. Blakslee, D. G. Proctor, E. J. Seldin, G. B. Spence, and T. Weng, J. Appl. Phys. 41, 3373 (1970).

${ }^{13}$ H. Ledbetter and S. Kim, Handbook of Elastic Properties of Solids, Liquids, and Gases (Academic, San Diego, 2001), Vol. 2.

${ }^{14}$ S. Baroni, A. D. Corso, S. de Gironcoli, and P. Giannozzi, http:// www.pwscf.org/.

${ }^{15}$ P. Hohenberg and W. Kohn, Phys. Rev. 136, B864 (1964).

${ }^{16}$ W. Kohn and L. J. Sham, Phys. Rev. 140, A1133 (1965).

${ }^{17}$ B. B. Pate, Surf. Sci. 165, 83 (1986).

${ }^{18}$ S. Iarlori, G. Galli, F. Gygi, M. Parrinello, and E. Tosatti, Phys. Rev. Lett. 69, 2947 (1992).

${ }^{19}$ A. Scholze, W. G. Schmidt, and F. Bechstedt, Phys. Rev. B 53, 13725 (1996).

${ }^{20}$ L. Fast, J. M. Wills, B. Johansson, and O. Eriksson, Phys. Rev. B 51, 17431 (1995).

${ }^{21}$ See EPAPS Document No. E-APPLAB-94-051905 for how to determine the elastic constants of hexagonal material using ab initio calculation. For more information on EPAPS, see http://www.aip.org/pubservs/epaps.html. 